CORRECTION

https://doi.org/10.1038/s41586-018-0294-9

\title{
Author Correction: Pancreas
} regeneration

Qiao Zhou \& Douglas A. Melton

Correction to: Nature https://doi.org/10.1038/s41586-018-0088-0, published online 16 May 2018.

In this Insight Review, '1989' should have been '1998' in the sentence "This deep understanding of pancreatic development was put to the service of regenerative medicine in 1989, when human embryonic stem cells (hES cells) were successfully cultured and opened the door to developing methods of deriving pancreatic islets from hES cells ${ }^{66}$.. This error has been corrected online. 